\title{
Hem-o-Lok polymer clips for major vascular control in paediatric minimally invasive surgery
}

\author{
Verity Haffenden $^{1} \cdot$ Robert T Peters $^{1} \cdot$ David Wilkinson $^{1} \cdot$ Nick Lansdale $^{1,2} \mathbb{C}^{\circ}$
}

Received: 4 November 2020 / Revised: 14 December 2020 / Accepted: 25 January 2021 / Published online: 4 March 2021

(c) The Author(s) 2021

\begin{abstract}
Purpose The challenge of endoscopic large vessel control has brought sweat to the brow of many a surgeon, yet the optimal method for use in a small working space remains unknown. Reports of delayed, major haemorrhage with exclusive energy device use have raised significant concerns. We, therefore, report outcomes of an alternative sealing device (Weck Hem-oLok polymer clips) in laparoscopic and thoracoscopic surgery.

Methods A retrospective review of all patients ( $<18$ years) who underwent laparoscopic splenectomy or thoracoscopic pulmonary resection between February 2018 and August 2020 (30 months) was undertaken. Data are presented as median (IQR). Results Thirty-three patients were identified (16 female); 16 underwent laparoscopic splenectomy and 17 thoracoscopic lobectomy/excision of congenital pulmonary airway malformation (CPAM) or resection of pulmonary sequestration. Age at surgery was 7 years 7 months (6 years 2 months -9 years 9 months) and weight $23.0 \mathrm{~kg}(20.2-37.4 \mathrm{~kg}$ ) in the splenectomy group: it was 16 months (13-19 months) and $12.0 \mathrm{~kg}(10.4-13.2 \mathrm{~kg})$ in the thoracic group. Hem-o-Lok clips (medium-large) were used to individually clip: (1) the main splenic artery and vein; or (2) pulmonary arterial branches and vein; or (3) systemic sequestration vessels, using a $5 \mathrm{~mm}$ endoscopic applicator. In most cases, two clips were placed proximally and one distally: the vessel then divided without energy. There were no incidences of clip failure during application or migration. There was no significant intra-operative or post-operative bleeding.

Conclusion Hem-o-Lok polymer clips are a safe and reliable method for major vascular control during endoscopic surgery in small children.
\end{abstract}

Keywords Laparoscopic $\cdot$ Thoracoscopic $\cdot$ Vessel ligation $\cdot$ Child $\cdot$ Splenectomy $\cdot$ Lobectomy

\section{Introduction}

Secure endoscopic vascular control is an integral technique for the safe practice of many paediatric surgical procedures, such as thoracoscopic pulmonary resections and laparoscopic splenectomy. Since the early 1990s, thoracoscopic pulmonary resections and laparoscopic splenectomy have been advocated over the open approach in paediatric surgery

Presented at the 20th BAPES Annual Meeting, November 5-6, 2020, London, United Kingdom.

Nick Lansdale

nick.lansdale2@mft.nhs.uk

1 Department of Paediatric and Neonatal Surgery, Royal Manchester Children's Hospital, Manchester, UK

2 Faculty of Biology Medicine and Health, The University of Manchester, Manchester, UK
[1-3]. Studies have demonstrated a reduction in post-operative pain and length of stay as well as cosmetic advantages $[4,5]$. However, the small working space of the paediatric patient complicates the already challenging task of dividing large vessels.

Methods of vessel sealing include suture ligation and staples/metal clips. Both these techniques are technically challenging in a small working space and using $5 \mathrm{~mm}$ paediatric ports and may slip/migrate. An alternative, and the preferred technique by many, is the use of advanced bipolar energy devices such as LigaSure (Medtronic), using bipolar and radiofrequency current with feedback mechanisms to allow for sealing of vessels without eschar formation. However, there have been anecdotal reports of delayed major haemorrhage after the exclusive use of energy devices for the sealing of major bloods vessels such as the splenic artery and vein. The Weck Hem-o-Lok (Teleflex) polymer locking ligation system provides an alternative, cold system minimising the 
use of thermal energy, and thus the risk of thermal injury to surrounding structures. The inner edge of the clip contains small teeth to prevent slipping and the applicator supplies tactile feedback to the surgeon when the clip is secure. Endoscopic appliers are available for the use of medium/large, large and extra-large clips: the medium/large size can be used on vessels up to $10 \mathrm{~mm}$ diameter and can be deployed using a $5 \mathrm{~mm}$ applier.

The optimal method of vascular control in the paediatric population is unknown. In this study, we share our outcomes with the use Hem-o-Lok clips during paediatric thoracoscopic and laparoscopic surgery to support a safe alternative to the use of energy devices for vessel sealing.

\section{Materials and methods}

A review was performed of all patients, under 18 years of age, who underwent a thoracoscopic resection of pulmonary sequestration, thoracoscopic lobectomy/excision of congenital lung lesion or laparoscopic splenectomy over a 32-month period from February 2018 to October 2020 at Royal Manchester Children's Hospital. Preliminary data were collected prospectively for this cohort of patients and medical records were reviewed if more detailed information was required.

Within the Department of Paediatric and Neonatal surgery at Royal Manchester Children's Hospital, there is a thoracic and upper gastrointestinal surgery team of three consultant paediatric surgeons and all operations were performed by one or more of these consultants.

Demographic data including age, weight and gender were collected as well as additional data to include diagnosis, surgical procedure, and details of surgical technique. In all patients, a $5 \mathrm{~mm}$ auto-applicator device was used to apply medium/large Hem-o-Lok clips via a $5 \mathrm{~mm}$ working port.

Outcome measures included intra-operative complications, post-operative complications, and length of inpatient stay. Continuous data were analysed and are presented as median and interquartile range (IQR).

\section{Results}

A total of 33 patients were included (16 female; 17 male) over the 32-month time-period. The standard approach was to apply two Hem-o-Lok clips proximally and a further one (two clips if the vessel was branching) distally then the vessel was divided without energy. In a small number of cases, a LigaSure device was used to seal and divide the distal vessel end only when there was adequate vessel length $(\sim 5 \mathrm{~mm})$ to apply the device away from the Hem-o-Lok clips remaining in the patient.
Laparoscopic splenectomy was performed in 16 patients. Age at time of surgery was 7 years and 7 months (6 years 2 months -9 years 2 months) and weight $23.0 \mathrm{~kg}$ (20.2-37.4 kg). Indications for laparoscopic splenectomy included hereditary spherocytosis $(n=10)$, HbSS sickle cell disease $(n=5)$ and transfusion dependent beta thalassaemia major $(n=1)$. Hem-o-Lok clips were used to individually seal the main splenic artery and splenic vein (Fig. 1a-c). Complications included one small wound haematoma and post-operative pancreatitis in one patient. There were no incidences of clip migration or failure, and no incidences of major intra-operative or post-operative haemorrhage. Postoperative length of stay was 2 days ( $2-3$ days). At the time of study, all 16 patients had outpatient follow-up post-surgery and all patients reported to be doing well with no surgical complications.

A total of 17 thoracoscopic resections were performed; 10 lobectomy/excision of congenital pulmonary airway malformation (CPAM) and 7 resections of pulmonary sequestrations. Age at surgery was 16 months (13-19 months) and weight $12.0 \mathrm{~kg}$ (10.4-13.2). Indications for surgery included intra-lobar sequestration [6], extra-lobar sequestration [1] and CPAM [10]. Hem-o-Lok clips were used to individually seal pulmonary arterial branches and the pulmonary vein or systemic sequestration vessels (Fig. 2a-c). Complications included one wound infection, one wound haematoma and one patient who required conversion to open thoracotomy due to failure to tolerate carbon dioxide insufflation. There were no incidences of clip migration or failure and no major intra-operative or post-operative bleeding. Post-operative length of stay was 2 days (2-2 days). At the time of study, 13/17 patients had outpatient follow up post-surgery. One patient was re-admitted post-operatively to their local hospital due to fever but was discharged after a 4-day course of antibiotics. All other patients reported to be doing well with no reported complications.

\section{Discussion}

This case series demonstrates a cohort of patients in whom Weck Hem-o-Lok clips were successfully used for vascular control without adverse effects. Although the literature currently contains little data on the optimal method of vascular control in the paediatric population, there are animal studies which have produced interesting data regarding 'burst pressure' with energy devices and clips. These studies demonstrate a significantly higher average burst pressure of $1200 \mathrm{mmHg}$ with Hem-o-Lok clips compared to $250 \mathrm{mmHg}$ with Ligasure [6]. However, we recognise these pressures are supra-physiological and suggest both techniques should provide adequate vessel sealing. 

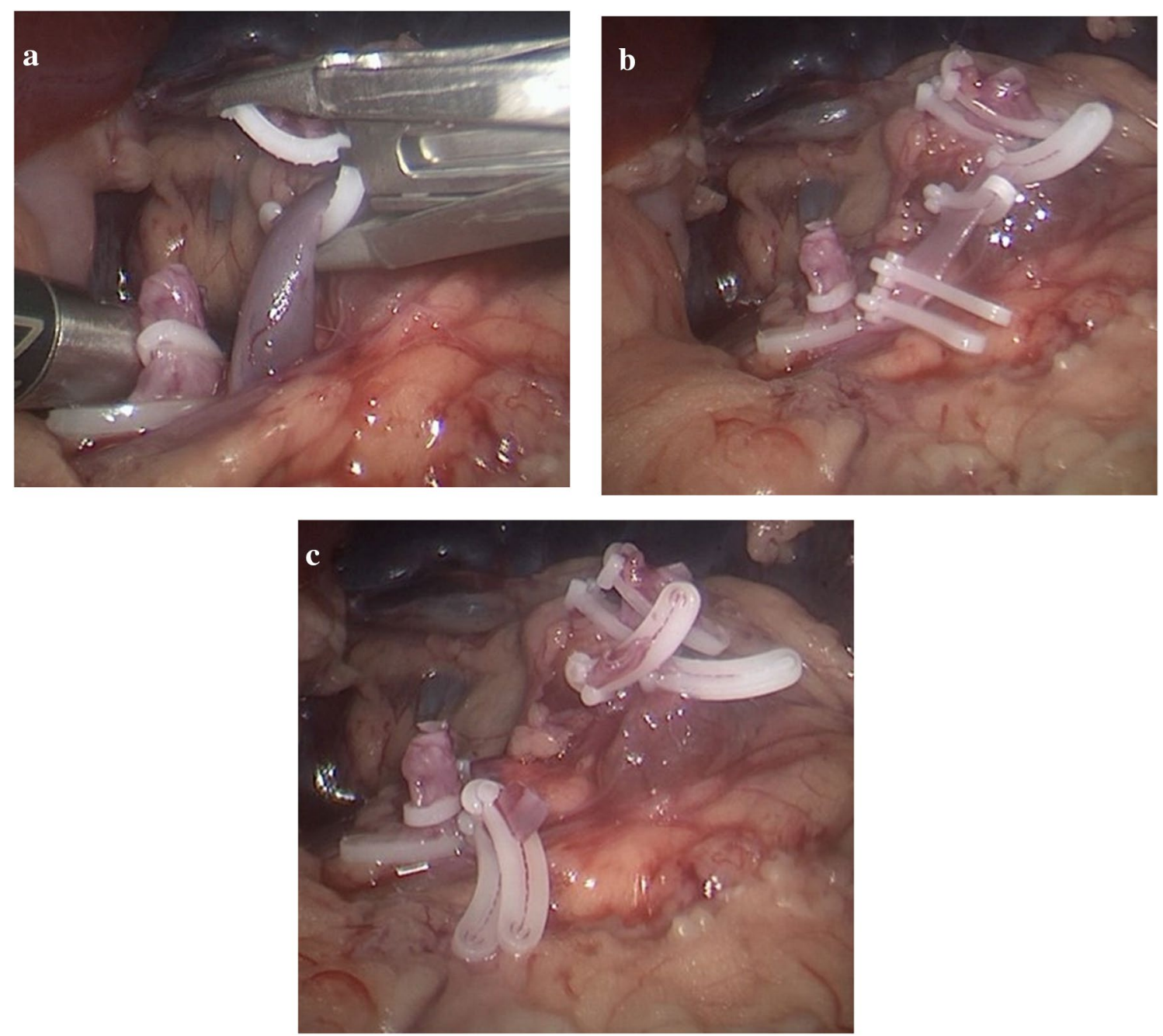

Fig. 1 a-c Laparoscopic splenectomy; a main splenic artery has been ligated. Hem-o-Lok clip is placed on main splenic vein b four Hem-o-Lok clips are placed $\mathbf{c}$ the vessel is divided between clips leaving two clips proximally

The patients in our study who underwent thoracoscopic resection had a median weight of $12.0 \mathrm{~kg}$ which demonstrates the small size of these patients and thus the small working space. Thermal injury is thus a reasonable concern with the use of energy devices which have shown a thermal spread of 1-3 $\mathrm{mm}$ [7]. The use of LigaSure to divide the vessel after clips have been applied is utilised by some [8] and may maintain the seal if the clip were to migrate. However, the potential for unrecognised thermal injury presents a concern of delayed major haemorrhage with potentially catastrophic consequences. The Hem-o-Lok clip is a cold sealing system presenting no risk of thermal injury when used without the concomitant use of an energy device.

The use of Hem-o-Lok clips is well described in the adult literature in procedures such as laparoscopic cholecystectomy and laparoscopic or retroperitoneoscopic nephrectomy
[9-12]. There are rare reports of clip migration into surrounding viscera $[13,14]$ which have not been documented in the paediatric population but should remain an important consideration. We acknowledge the short duration of follow-up to date but note that no patients in this study have reported symptoms post-operatively that could be attributable to clip migration.

\section{Conclusions}

This case series demonstrates that Hem-o-Lok polymer clips are a safe, reliable, alternative to the use of energy devices for major vascular control during minimally invasive surgery in small children. 

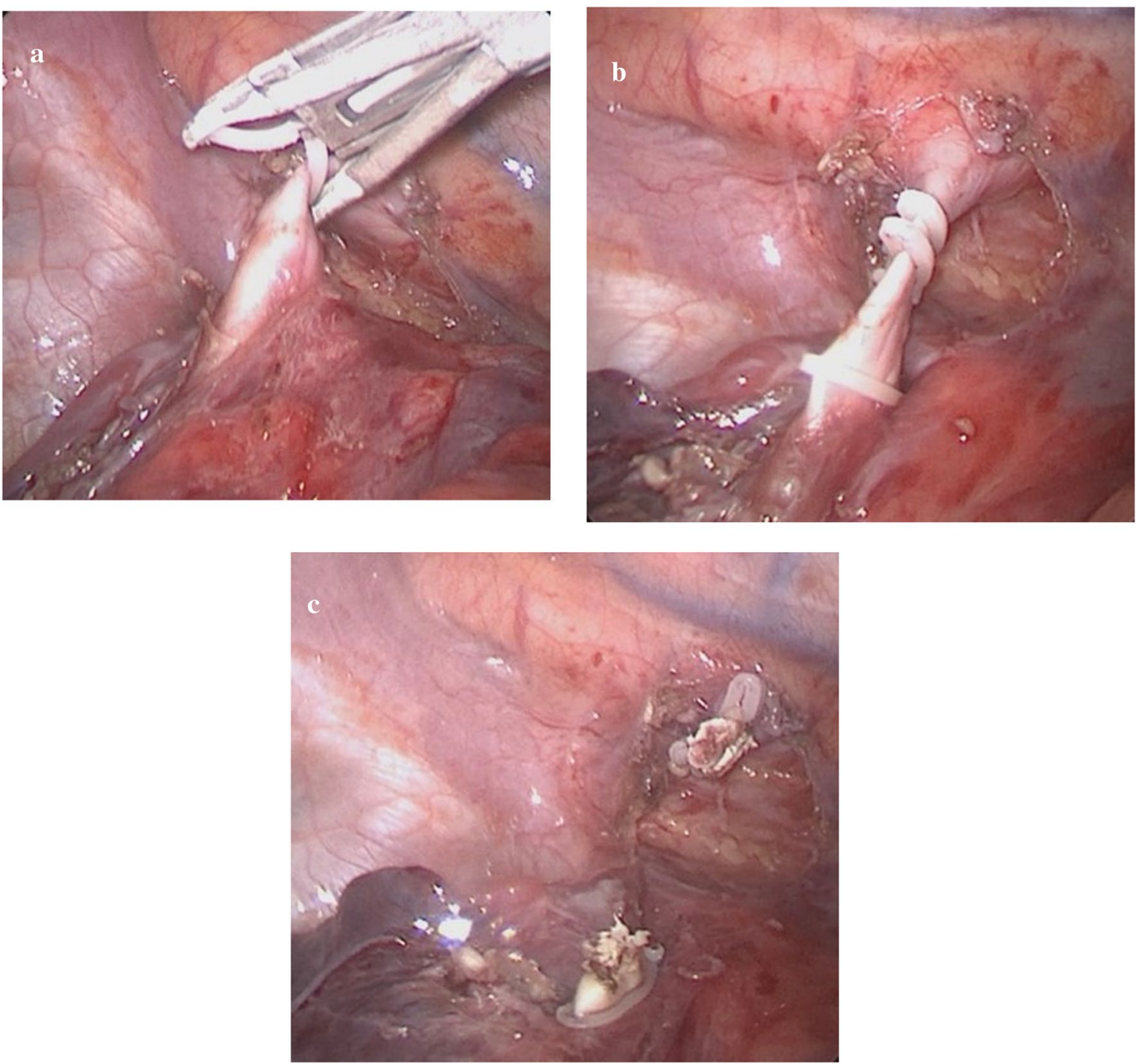

Fig. 2 a-c Thoracoscopic resection of pulmonary sequestration. a Hem-o-Lok clips are placed on the systemic sequestration vessel. b 3 Hem-oLok clips are placed. $\mathbf{c}$ The vessel is divided leaving two clips proximally

Author contributions Study conception + design-NL. Data acquisition- $-\mathrm{VH}$. Analysis + data interpretation-VH, NL, drafting of the manuscript-VH. Critical revision-NL, RP+DW.

Funding Not applicable.

Availability of data and materials Not applicable.

Code availability Not applicable.

\section{Compliance with ethical standards}

Conflict of interest The authors declare that they have no conflict of interest.

Open Access This article is licensed under a Creative Commons Attribution 4.0 International License, which permits use, sharing, adaptation, distribution and reproduction in any medium or format, as long as you give appropriate credit to the original author(s) and the source, provide a link to the Creative Commons licence, and indicate if changes were made. The images or other third party material in this article are included in the article's Creative Commons licence, unless indicated otherwise in a credit line to the material. If material is not included in the article's Creative Commons licence and your intended use is not permitted by statutory regulation or exceeds the permitted use, you will need to obtain permission directly from the copyright holder. To view a copy of this licence, visit http://creativecommons.org/licenses/by/4.0/.

\section{References}

1. Rothenberg SS (2000) Thoracoscopic lung resection in children. J PediatrSurg 35:271-275. https://doi.org/10.1016/S0022 -3468(00)90023-X

2. Polites SF, Habermann EB, Zarroug AE, Thomsen KM, Potter DD (2016) Thoracoscopic vs open resection of congenital 
cystic lung disease- utilization and outcomes in 1120 children in the United States. J PediatrSurg 51:1101-1105. https://doi. org/10.1016/j.jpedsurg.2015.12.004

3. Rescorla FJ, Engum SA, West KW, Scherer LRT, Rouse TM, Grosfeld JL (2002) Laparoscopic splenectomy has become the gold standard in children. Am Surg 68:297-301

4. Rothenberg SS, Middlesworth W, Kadennhe-Chiweshe A et al (2015) Two decades of experience with thoracoscopic lobectomy in infants and children: standardizing techniques for advanced thoracoscopic surgery. J LaparoendoscAdvSurg Tech 29:423-428

5. Murawski M, Patkowski D, Korlacki W, Czauderna P, Sroka M, Makarewicz W et al (2008) Laparoscopic splenectomy in children-a multicenter experience. J PediatrSurg 43:951-954. https://doi.org/10.1016/j.jpedsurg.2007.11.040

6. Elliott SP, Joel AB, Meng MV, Stoller ML (2005) Bursting strength with various methods of renal artery ligation and potential mechanisms of failure. J Endourol 19:307-311. https ://doi.org/10.1089/end.2005.19.307

7. Landman J, Kerbl K, Rehman J, Andreoni C, Humphrey PA, Collyer W et al (2003) Evaluation of a vessel sealing system, bipolar electrosurgery, harmonic scalpel, titanium clips, endoscopic gastrointestinal anastomosis vascular staples and sutures for arterial and venous ligation in a porcine model. J Urol 169:697-700. https ://doi.org/10.1016/S0022-5347(05)63995-X

8. Bignon H, Buela E, Martinez-Ferro M (2010) Which is the best vessel-sealing method for pediatric thoracoscopic lobectomy? J LaparoendoscAdvSurg Tech 20:395-398. https://doi.org/10.1089/ lap. 2010.0010
9. Liu Y, Huang Z, Chen Y, Liao B, Luo D, Gao X et al (2018) Staplers or clips? A systematic review and meta-analysis of vessel controlling devices for renal pedicle ligation in laparoscopic live donor nephrectomy. Medicine (United States) 97:e13116. https:// doi.org/10.1097/MD.0000000000013116

10. Aminian A, Khorgami Z (2012) Hem-o-Lok Clip is safe in minimally invasive general surgery: a single center experience and review of data from food and drug administration. J Minim Invasive Surg Sci 1:52-57. https://doi.org/10.5812/jmiss.1885

11. Aminian A, Karimian F, Toolabi K, Mirsharifi R (2010) Application of Hem-o-Lok clip in basic laparoscopic procedures: a single center experience on 856 cases and review of data from food and drug administration. SurgEndosc Other Interv Tech 24:5501-5502

12. Simforoosh N, Sarhangnejad R, Basiri A, Ziaee SAM, Sharifiaghdas F, Tabibi A et al (2012) Vascular clips are safe and a great cost-effective technique for arterial and venous control in laparoscopic nephrectomy: single-center experience with 1834 laparoscopic nephrectomies. J Endourol 26:1009-1012. https:// doi.org/10.1089/end.2011.0619

13. Yu CC, Yang CK, Ou YC (2015) Three types of intravesical Hem-o-Lok clip migration after laparoscopic radical prostatectomy. J LaparoendoscAdvSurg Tech 25:1005-1008. https://doi. org/10.1089/lap.2015.0150

14 Barabino M, Luigiano C, Piccolo G, Pellicano R, Polizzi M, Giovenzana M et al (2019) Hem-o-Lok clip migration into the duodenum after laparoscopic digestive surgery: a systematic review. Minerva Chir 74:496-500. https://doi.org/10.23736/S0026 $-4733.19 .08152-5$ 